\title{
MODELS OF LARGE-SCALE E-LEARNING
}

\author{
Martin Weller \\ The Institute of Educational Technology \\ The Open University \\ United Kingdom \\ Email: m.j.weller@open.ac.uk
}

\begin{abstract}
Early interest in e-learning focused around the possibility of large-scale courses. This led to pronouncements of the demise of the educator, which were based on an infinite lecture hall pedagogy. However, cost-effective models of large-scale e-learning have proven difficult to implement. This paper examines some of the initial reaction to the notion of large-scale courses and sets out the cost difficulties associated with such courses. Five models of large-scale e-learning are proposed. Each of these have implicit associated pedagogies. The majority of these assume instructivist pedagogy. Large-scale models that use a more constructivist pedagogy may be possible using community based approaches. The importance of differentiating between pedagogic styles and scale of implementations is stressed as it highlights the reasoning behind some of the initial claims against e-learning.
\end{abstract}

\section{KEYWORDS}

Scalability, instructivist pedagogy, constructivist pedagogy, community, e-learning

\section{INTRODUCTION}

One of the characteristics of online learning that initially caused a good deal of interest was its potential to teach large numbers of people. Whereas traditional education methods are limited by physical constraints, such as the size of any given lecture hall, the virtual world has no such limits. With sufficient server bandwidth, then any number of people can view a streamed video lecture at any time. For universities, training centers, or colleges struggling to cope with increased student numbers and rising real estate prices, scaling seems an attractive solution.

Similarly, whereas physical resources are limited, digital resources are limitless, since they can be reproduced easily (and perfectly). Electronic access to journals is one means of making scarce resources more widely available. Access to laboratory and expensive equipment is another area where there are often restrictions that can inhibit the learning experience of individuals. Virtual laboratories, simulations, and remote access to real equipment are all means of alleviating resource issues.

Hopes were often based on the idea that one lecturer could effectively educate a thousand students, as well as ten students, or that once lectures were recorded and stored, there would be no need for the lecturer. This naturally made educators anxious that they would be superseded by technology. This anxiety and viewpoint is evident in the following statements:

"Once the faculty converts its courses to courseware, their services are in the long run no longer required. They become redundant, and when they leave, their work remains behind. In Kurt Vonnegut's classic novel Player Piano the ace machinist Rudy Hertz is flattered by the 
automation engineers who tell him his genius will be immortalized. They buy him a beer. They capture his skills on tape. Then they fire him. Today faculty are falling for the same tired line, that their brilliance will be broadcast online to millions” [1].

"A curriculum, once created, could be offered electronically not just to hundreds of students nearby but to tens of thousands around the world" [2].

As well as being technologically deterministic and dystopian in outlook, what these quotes have in common is that they view the educator's content expertise as their prime, even sole, asset. Once this is extracted, then the educator has no remaining value. However, the process of extracting knowledge was happening long before the arrival of the Internet. It is precisely the reason behind the publication of books and journal articles - to disseminate and share knowledge. The publication of books by experts does not render that expert redundant—in fact, it usually increases their worth.

The educator's role is to teach and to add value to content. The same applies in online courses. Most educators will find that their skills are required just as much, if not more, when they move online. Eminent academics may indeed prefer to produce good course materials and then leave the delivery and support of this material to other staff, while they use their time in research, returning periodically to update the course.

An infinite lecture hall view of online teaching is only really possible if there is little student support needed. This may well be suitable for some topics. For instance, web-based training courses in IT might work well as stand-alone products with a good mix of media to explain the concepts. There is little evidence, however, that higher education students or organizations wishing to develop complex skills would be content with such an approach.

Many of the prophecies regarding the redundancy of educators for large-scale online courses were based on transferring the teaching model of face-to-face to the online environment. In this scenario, overcoming the physical restrictions of class size and recording lectures would inevitably lead to large student classes

and instructor redundancy. However, most educators have quickly discovered that simply transferring their teaching approach from one medium to the other is not effective. For a variety of reasons, the standard lecture is not an effective online teaching method. Most educators therefore find themselves adapting their approaches, providing more support and being more involved with students, not less. The restrictions on the student-to-educator ratio remain, and larger class sizes do not become immediately feasible. Similarly, good educators are just as highly valued online, so they are not removed from the educational process. If anything, their significance in achieving student satisfaction is made greater because of the nature of the online course.

For the purposes of the discussion that follows, let us define a large-scale course as one which cannot be supported by a single academic, or by an academic with one or two part-time staff. Student numbers will likely be in the hundreds or thousands in such courses. These are courses (or more generically, learning experiences) where the numbers of learners require course designers to rethink the standard support model, and the relationship between the educator and learner is necessarily altered.

\section{COST IMPLICATIONS}

One of the motivating factors for senior management to drive the uptake and development of e-learning 
initiatives has been the potential cost benefits they offer. As we have discussed above, the notion of an infinite lecture hall, or increased student-to-tutor ratios, is not as easily realized as many first believed. The cost benefits of creating large population courses are not as great as once envisaged, and indeed may be a limiting factor because of the difference between fixed and variable costs in course production and delivery.

Traditional distance education technologies have high fixed costs but relatively low variable costs. That is, the initial production cost is high, but then the price per student is relatively low. For instance, a television program or video is costly to produce, requiring expertise in scripting, camera operators, lighting, sound, and editing, as well as the use of expensive equipment. Plus, programs are often filmed in a variety of locations with all the attendant travel and subsistence costs. However, once a program is made, it is relatively cheap to reproduce (for example, by using video cassettes), so the costs do not increase greatly with the number of students. Such high fixed costs require a number of students to reach a break-even point and are well suited to large population courses which run for several presentations.

Variable costs, on the other hand, are those that increase with the number of students. For example, the payment of part-time tutors does not achieve economies of scale - the larger the population of a course, the greater the number of tutors required. Computer-mediated communication (CMC) (in any form, but let us assume the most common type of asynchronous text-based conferencing) is the basis for most online courses. It requires moderators to promote student dialogue in various discussions areas, tutors to run and support collaborative activities, and experts to provide help and advice in support conferences. These all represent variable costs. The degree to which these are an integral part of the course will depend on the course design and pedagogy. A constructivist approach that promotes dialogue, collaboration and student guidance will rely heavily on computer conferencing; indeed, some would argue that this dialogue is the course. Such an approach therefore carries a high variable cost component [3].

Bates analyzed the cost structure of several educational technologies [4]. He used cost per student study hour to provide a comparable measurement between technologies. This is specified as cost per student study hour = total cost of materials / (number of hours spent studying the material times total number of students over the life of the course). Bates produced several graphs illustrating these costs, over an eightyear period. Two of these are shown below:

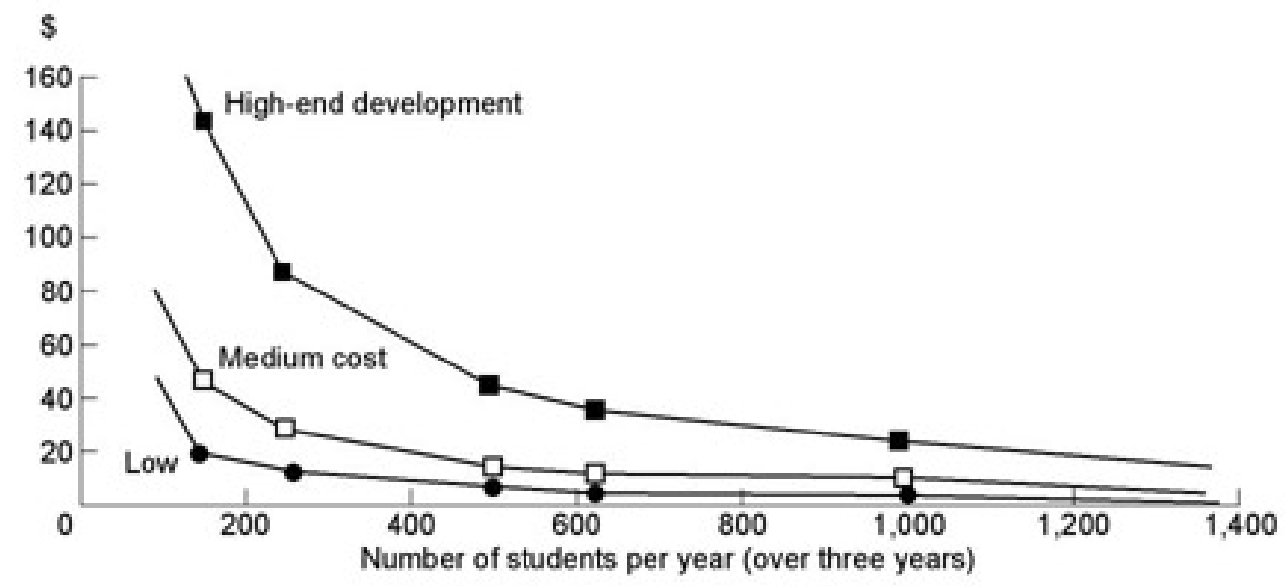

Figure 1: The costs for computer based learning on a distance education course (after Bates 1996) 


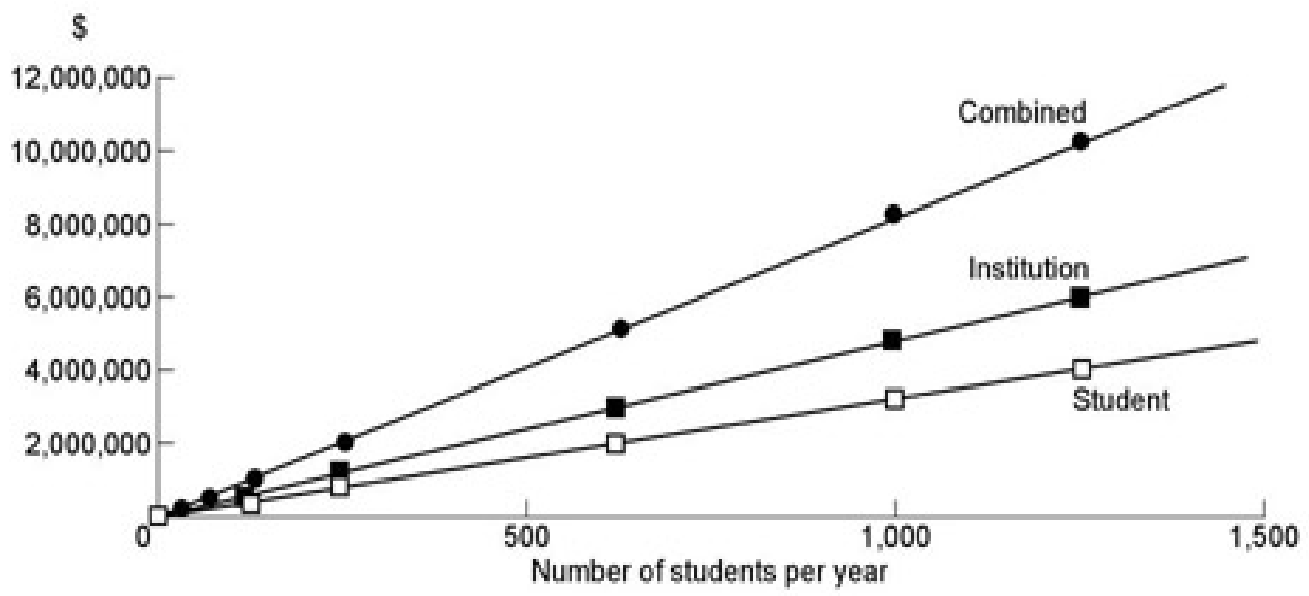

Figure 2: The costs for computer mediated communication (CMC) on a distance education course (after Bates 1996)

The graph for computer-based learning (CBL) shows three scenarios: high-end development, such as inhouse, bespoke developed multimedia; medium-end development, which might be realized through working with a software consultancy firm; and low-end development, which represents uncustomized 'off-the-shelf' products. All three scenarios follow the same pattern: they have high initial, fixed costs associated with development or purchase, but low variable costs, making them relatively cheap as student numbers increase.

The CMC graph shows a different pattern. The CMC graph divides the costs between those associated with the institution and those associated with the student, as well as a combined cost. Here the costs increase proportionally as student numbers increase. Bates claims that "total costs rise directly with student numbers [4]. There is relatively little front-end investment in these courses, and if interactivity, a major feature of CMC, is to be effective, the number of instructors increases with the number of students. Instructors are the main cost with this technology” [4, p. 223].

In effect, these two graphs represent the different views of e-learning. Many of the views expressed above that claim e-learning will make educators redundant and will lead to the commoditization of knowledge are based on the CBL cost model. The proponents of e-learning who claim that it requires a great deal of educator input, and thus enhances the educator role, are operating with the CMC costing model.

Many institutions are now struggling with balancing these two, and often combining them. Many online courses have both high fixed costs, for instance in producing online CBL, and also high variable costs because they have a strong CMC model. One possible solution is to develop partnerships and coalitions, so that universities or e-learning providers pool their resources, for example using learning object repositories. This enables them to share expertise and cost in creating effective e-learning material. Because of the digital nature of this material it can be easily reused, adapted and customized by each partner, and used in different contexts. Whether such approaches become commonplace and are really scalable to large populations remains to be seen.

\section{MODELS OF LARGE-SCALE COURSES}

Having dismissed the initial response to creating large-scale courses as being somewhat naive about 
pedagogy and customer demand, let us now look at how such courses might be realized. Five main strategies for creating large-scale courses can be identified. The models are the main approaches to realizing large-scale courses. The choice of model will be influenced by a number of factors, including the resources available, the intended audience, and the pedagogical requirements. Thus, the learning experience will be different in each of the models; they should not be viewed as alternative approaches to the same learning goal. For example, the low support model will provide a largely individual, instructivist experience, whereas the community model will support a more collaborative, informal learning experience.

\section{A. Low Support}

In the low-support model, students have little or no contact with an educator. They either study the online material individually, or they have access to a peer group. To work effectively, the material needs to be of a high quality, usually containing a variety of media. It is better suited to some markets and topics, such as some web-based training material in the IT area, where the user simply wants to develop a new skill without engaging with an educator or a peer set. Feedback will often be automatic, realized through multiple choice questionnaires, intelligent tutoring agents, and system-tracking software to monitor student behavior and provide help and advice. While support can also be offered via large-scale CMC, with one or two moderators dealing with queries, or via telephone call centers, the student does not have a dedicated tutor with whom ongoing interaction is an integral part of the course design. The sort of training material delivered by many organizations is an example of the low-support model. The mobile phone company Nokia plan to deliver e-learning to 200 million people throughout their supply chain [5]. Obviously, such figures can only be achieved with no or little live support. The e-learning products that Nokia use are usually small pieces of Flash animation, video, games, simulations, manuals etc.

This approach has similarities with the emporium model proposed by Twigg [6], which is "heavily dependent on instructional software, including interactive tutorials, computational exercises, electronic hypertext books, practice exercises, solutions to frequently asked questions, and online quizzes.”

\section{B. Hierarchical Support Structures}

The hierarchical-support-structures model follows the traditional distance-education model. In this model, the students have specifically designed course materials (which may be all online or a mixture of online, print, and other media). They are supported by part-time tutors. The central course team responsible for the course supports the tutors. This has many of the high production costs of a traditional distanceeducation course and the high variable costs of a richly supported CMC course. An example is the UK Open University's level 1 course titled "You, Your Computer and the Net," which had around 10,000 students. Weller and Robinson detail a hierarchical model of student support via CMC [7]. However, it remains unclear whether this model is cost-effective in distance education, as it represents a potential combination of high fixed and high variable costs. Twigg [6] reports considerable savings in the redesign of blended campus-based courses, which often adopt this strategy, as it makes use of "alternative staffing," replacing "expensive labor (faculty and graduate students) with relatively inexpensive labor (undergraduate peer mentors and course assistants) where appropriate.”

\section{Expert Plus Part-Time Support}

The expert-plus-part-time-support model has some characteristics of the large lecture hall approach. This model has the expert, whose opinion is highly valued, but whose time is also very precious, possibly providing lectures (either online or face to face) or study material. There is little or no contact between 
students and the expert, but the students are supported by part-time tutors online, who aid their study of the material and deal with queries, offer guidance, etc. As with the previous model, there may be some hierarchical structure, with tutors having access to the expert for queries they cannot answer. Having captured the expert in some format, then the resource may be used on different courses or over successive years. This is the type of model many of those apprehensive about online education have in mind, but it is most likely to be successful when the experts themselves do not have time to teach or support students. If the expert-plus-part-time-support model was an adaptation of the existing distance-education model to the online medium, then this model can be seen as an adaptation of the traditional campus-based lecture model. In terms of support and approach, it lies between the expert-plus-part-time-support model and the hierarchical-support-structures model.

The expert-plus-part-time-support model may be attractive where the expert is in high demand outside of education, in professional vocational subjects such as law, medicine, and business. It is also likely to be adopted as a response to increasing student numbers and lower educational resources, such as in largescale foundation classes that incorporate face-to-face lectures from academics and online support from postgraduate students. The model can be enhanced beyond the standard lecture plus support by the incorporation of interactive elements, multi-media elements, automated assessment, and more. With this additional content, the online model begins to offer more than its face to face counterpart.

\section{Automatic Creation of Personalized Courses}

By using a learning-object approach, it is feasible to create courses on the fly that will be well suited to the individual learner. These are assembled from a vast database of such resources and compiled into a single course. These resources may have been produced through collaboration or partnership with other institutions. Such courses may vary in their time span from a few minutes to several hours or days. They will be better suited to learning within the workplace and are well suited to a just-in-time approach. They require a large population of learning objects to draw from initially, if they are to be successful. There has been very little work done in this area. Investigations into whether it represents an effective means of delivering courses on a large scale are sparse.

This is the ultimate aim of many who are promoting the learning-objects approach. If there is a sufficiently populated pool of reusable digital objects, which are appropriately marked with metadata, then the idea is that these can be automatically assembled to meet the needs of an individual learner. For example, Lave and Wagner [8] state that "while training company providers are also engaged in this reuse activity, they are aiming at what they perceive to be a much bigger market: content aggregation on the fly by individual learners or training providers.”

In this scenario, learners receive an individual experience based on their learning profiles, as derived from diagnostic tests, learner preferences, immediate learning demands, and so on. This large-scale delivery, but individualized content, is similarly put forward by Advanced Distributed Learning consortium (ADL), an organization that is devising learning object approaches for the US military and has developed the SCORM specification (Sharable Content Object Reusable Model). ADL states on its web site [9] that:

"The purpose of the ADL Initiative is to ensure access to high-quality education and training materials that can be tailored to individual learner needs and made available whenever and wherever they are required.”

"The ADL Initiative is designed to accelerate large-scale development of dynamic and costeffective learning software and systems to stimulate an efficient market for these products in 
order to meet the education and training needs of the Military Services and the nation's workforce of the future. ADL will achieve this through the development of a common technical framework for computer and net-based learning that will foster the creation of reusable learning content as instructional objects.”

\section{E. Peer-Supported Community Models}

Peer-supported-community courses take advantage of their large populations by promoting dialogue and support between students. In this respect, students themselves perform many of the functions of the educators. For instance, peer assessment or evaluation might be an effective way of providing feedback on student's work without the educator reviewing all assessment. However, many such courses do require a good deal of educator input to provide guidance and support, so they may not offer a cost-effective means of increasing scale.

Within organizations, informal learning approaches that promote dialogue and exchange between employees can be an effective way of disseminating information without the need for formal training courses. Such communities often arise outside of formal organization boundaries and may be encouraged by professional bodies.

Such an approach has gained a good deal of credence with the notion of communities of practice [10, 11]. For example, Lesser and Everest [12] claim that

"These communities of practice, (or COPS), that exist in formal organizational structures play a key role in ensuring that the intellectual capital in an organization is created, shared, and used effectively. Organizations, ranging from the World Bank to IBM, have begun to actively foster and support these communities so that they can more effectively take advantage of their knowledge assets.”

Gongla and Rizzuto [13] detail the development of 60 "knowledge network communities" in IBM that covered the various countries in which they have a presence and the numerous roles within the organization. This clearly allows learning to take place on a large-scale across the organization.

\section{ASSOCIATED PEDAGOGIES}

Most of the models outlined above have implicit assumptions regarding the pedagogies that best suit them. It is therefore important to both appreciate this and to consider what pedagogical approach best meets the needs of a particular set of learners, to determine the approach that allows the model to achieve its scale. For example, the low-support model is largely suited to a didactic or instructivist approach, which leads the learner through concepts and set activities. It may make use of quizzes and self-reflection, but there is little scope for dialogue or collaboration. However, increasingly there may be potential for constructivist approaches based on games, simulations and role-playing environments, which do not require high intensity support from educators, but offer a rich and varied experience. These are often expensive to develop, however, and are still largely unproven in the educational arena. So, for the foreseeable future, the majority of courses adopting this model will remain instructivist in design.

The hierarchical-support-structures model is probably the most pedagogically neutral of the models outlined. It can incorporate collaborative activities that are supported by tutors, and it can be straightforward instructional material with tutor support and assessment feedback, or a mixture of these, 
and so on. Managing this support so that all learners receive an individual and quality learning experience is the main challenge with this model, as it relies heavily on the quality of the tutor support to succeed. It therefore requires large-scale induction, training, and monitoring procedures, which are costly to implement.

The expert-plus-part-time-support model necessarily has a focus on an individual expert, so it will tend to be instructivist, being a variation on the traditional lecture. There is the potential for some variation in the follow-up work that is supported by part-time staff, such as collaborative activities to explore the issues raised by the expert. However, such a model is usually seen as a means of maximising the expert's availability and solving resource problems, rather than developing innovative teaching solutions. So, it is likely that the instructional approach will predominate.

Although many proponents of learning objects claim that they are pedagogically neutral, the sort of automated course outlined in the learning-object approach can only really operate if, once again, an instructional pedagogy is practised. The main thrust of this approach is that it operates by having an individualized experience, with learners studying just-in-time. There is therefore little room for collaboration or dialogue in such a model. This is only one realization of learning objects, and it is important to differentiate the SCORM approach from others. Dan Rehak, one of the leading architects of the learning object approach has said "SCORM is essentially about a single-learner, self-paced and selfdirected. It has a limited pedagogical model unsuited for some environments... SCORM has nothing in it about collaboration. This makes it inappropriate for use in higher education and K-12" [14]. Although some dispute these claims, a large-scale implementation model based on the automatic assembly of such objects can only really function if it is based around a single-learner with largely instructivist material.

The peer-supported-communities model is perhaps the most interesting from a pedagogical perspective. Such approaches make significant use of informal learning and so are outside the scope of much of the formal education and training focus. However, it may be that it is only through the fostering of such approaches that large-scale e-learning can be implemented in a manner that is supportive and interactive for the individual.

\section{DISCUSSION}

Large-scale e-learning can be achieved, although it is not as simple or as cost-effective as once imagined. The models above demonstrate what a broad umbrella term 'e-learning' is, since it can refer to a range of learning experiences - from watching a 2 minute animation to studying a nine months master's course based on constructivist principles. If one considers not just large-scale implementations, then the term also encompasses small courses, blended courses, informal learning through dialogue, and so forth. For this reason, it may not be a useful term as it focuses on the medium. Given such a variety of learning experience, this is perhaps the least significant factor. A normal campus-based undergraduate lecture class has more in common with the expert-plus-part-time-support model than this does with peer-supportedcommunity model, say. So, to group them all simply as e-learning may not be helpful. An alternative approach may be to group learning experiences according to pedagogy.

The focus of these models has been at the course level as this is largely the level at which resource and attention in e-learning is applied. However, it should be noted that courses do not exist in isolation, usually forming part of a curriculum of study or a professional development program. The learner experience across the whole program should also be borne in mind. For example, large enrollment foundation courses may be delivered in this manner, while smaller scale electives operate on a different model. Across the program as a whole, the learner may then experience a range of pedagogies and 
develop a range of skills. Thus, the expert-plus-part-time-support model may be suitable for a compulsory course with a large cohort, and a more collaborative approach may be used on a lower population course.

What the models above also demonstrate is that, on the whole, large-scale models tend to be largely instructivist. While it is possible to achieve instruction on a large-scale, other pedagogical approaches that focus on creating an individual experience, using dialogue and learning through collaboration, require intensive support and may be more difficult to realize. Detractors of e-learning have often been confused by this, assuming that all e-learning has to be large-scale, and therefore decrying it as only relevant to training. For example, Noble [15] claims that "education must be distinguished from training (which is arguably more suitable for distance delivery), because the two are so often conflated.”

Intimate, constructivist courses are possible and working well in e-learning, but these approaches may not be scalable. Only the second and last of the models outlined offer any potential for moving away from an instructivist model. For many e-learning practitioners, a constructivist approach is essential in achieving a quality learning experience. For example, Beaty, Hodgson, Mann, and McConnell [16] set out a networked e-learning manifesto for higher education in which they state

"Educational values which contribute to quality in learning and teaching environments are those that seek to encourage dialogue, exchange of ideas, intrinsic approaches to study and engagement. It is this that we need to support through networked e-learning.”

Beaty and colleagues feel the need to expand the e-learning term to 'networked e-learning' to partly differentiate between the sorts of e-learning used in training and address the problem of an over-general term outlined above. For Beaty and colleagues, it is questionable whether e-learning, as they define it, can ever be realized on a large scale. The only approaches that might meet their needs are the hierarchicalsupport-structures model and the peer-supported-community model. For example, they go on to say:

"In our view of networked e-learning, the relationship between teachers and learners is based on collaboration and co-construction of knowledge rather than on that of expert and acolyte. Such a view of the relationship between learners and teachers is one that is supported by the idea of the learning community."

\section{SUMMARY}

This paper has largely focused on the presentation costs of large-scale e-learning and how these can be alleviated through different models of support. The overall cost of a course is determined not just by the presentation costs, but also the production costs. The potential for reusing and sharing digital resources in the form of learning objects is potentially a solution to reduce these costs. However, both aspects of the cost equation need to be addressed, and the costs associated with presentation need to be carefully considered and fully understood.

While large scale e-learning is most easily realizable with low support and relatively instructivist material, these approaches often have low retention. More supported, engaging courses are necessary to fulfill the potential of e-learning as both a delivery mechanism for a wide range of resources and interactive media and a communication medium. Further investigation is required to ascertain whether large-scale learning, which combines engaging content and intensive support can remain cost-effective. The use of peer-supported communities is probably the best solution for realizing large-scale implementations, combined with a hierarchical-support model. However, both costs and quality of learning in such a hybrid approach would need to be carefully monitored. 


\section{REFERENCES}

1. Noble, D. Digital Diploma Mills, Part I: The Automation of Higher Education. FirstMonday 3 (1): 1997. Available Online: http://www.firstmonday.dk/issues/issue3_1/noble/.

2. Noam, E. Electronics and the Dim Future of University Science 270: 247-249, October 13, 1995. Available Online: http://www.asis.org/annual-96/noam.html.

3. Annand, D. The Problem of Computer Conferencing for Distance-based Universities. Open Learning 14 (3): 47-52, 1999.

4. Bates. A. W. Technology, Open Learning and Distance Education. Routledge: London, UK, 1995.

5. Van Hooydonk, S. Offering E-learning Throughout the Customer Cycle: Case Nokia. Elearn international conference, Edinburgh, February $18^{\text {th }}-19^{\text {th }} 2003$. Available Online: http://www.elearninternational.co.uk/speaker_presentations.asp.

6. Twigg, C. A. Improving Learning and Reducing Costs: New Models for Online Learning. Educause Review 38: 28-38, September/October 2003.

7. Weller, M. J. and Robinson L. Scaling Up an Online Course to Deal With 12,000 Students. Education, Communication and Information 1 (3): 307-323, 2002.

8. Rehak, D. R. and Mason, R. Keeping the Learning in Learning Objects. In Littlejohn, A (ed.) Reusing Educational Resources for Networked Learning. Kogan Page: London, 2003.

9. Advanced Distributed Learning. Available Online: http://www.adlnet.org/index.cfm?fuseaction=abtadl.

10. Lave, J. and Wenger, E. Situated Learning: Legitimate Peripheral Participation. Cambridge University Press: Cambridge, 1991.

11. Wenger, E. Communities of Practice: Learning, Meaning, and Identity. New York: Cambridge University Press, 1998.

12. Lesser, E. and Everest, K. Using Communities to Practice to Manage Intellectual Capital. Ivey Business Journal 65 (4): 2001. Available Online: http://www.iveybusinessjournal.com/archives/default.asp?intYear=2001.

13. Gongla, P. and Rizzuto, C. Evolving Communities of Practice: IBM Global Services Experience. IBM Systems Journal 40 (4): 842-862, 2001.

14. Kraan, W and Wilson, S. Dan Rehak: SCORM is Not for Everyone. CETIS, October 02, 2002. Available Online: http://cetis.ac.uk/content/20021002000737.

15. Noble, D. Technology and the Commodification of Higher Education. 2002. Available Online: http://www.monthlyreview.org/0302noble.htm.

16. Beaty, E., Hodgson, V., Mann, S. and McConnell, D. Working Towards E-Quality in Networked E-Learning in Higher Education: A Manifesto Statement for Debate. Presented at Understanding the Implications of Networked Learning for Higher Education Seminar Series. University of Sheffield, Sheffield. March 26, 2002.

\section{ABOUT THE AUTHOR}

Dr. Martin Weller is a Senior Lecturer in the Institute of Educational Technology at the Open University in the UK, and the Project Director for the Open University's VLE. He is the author of numerous articles on e-learning and the book "Delivering Learning on the Net." His research interests are in the implications of learning objects, deployment of Learning Design, and open architecture learning environments. 